\title{
THE DYNAMICS OF LIVING AND THINKING SYSTEMS, BIOLOGICAL NETWORKS, AND THE LAWS OF PHYSICS
}

\author{
V. GONTAR
}

Received 23 January 2004

\begin{abstract}
"In order to make fundamental progress, we needed to introduce new physical concepts, such as deterministic chaos and Poincaré resonances, and new mathematical tools to turn these weaknesses into strengths."
\end{abstract}

Ilya Prigogine, (1997)

Discrete chaotic dynamics (DCD) of living and thinking systems are presented in a form of networks of interacting agents with the abilities of energy and information exchange. Special dynamical principles followed by the systems of basic discrete time and space difference equations are introduced. Emergent, self-organized behavior of complex living and thinking systems is presented by the different patterns generated by the DCD algorithms. Artificial life and brain systems based on DCD principles and mathematical models are proposed.

\section{Introduction}

In the last few decades, the constant technical development and the tremendous effort in the study of living systems have made it possible to accumulate massive experimental results on living cells, such as genomic sequences, protein structures, and the signaling pathways and regulatory mechanisms of the cycles of living cells. How to make all this data observable and verifiable is the problem of theoretical biology; it still inspires more questions than answers. Composed of atoms and molecules, living cells hardly obey the physical laws of quantum mechanics and statistical physics. On the scale of their operations, living cells also do not obey the laws of thermodynamics. Numerous attempts to depict the dynamics of living cells with the laws of physics have not allowed biologists to understand any better what they investigate. The extreme complexity of the structural and behavioral properties of living cells does not manifest any dynamics similar to that evidenced in the successful study of inert matter in physics. Even an individual living cell presents behavior comparable to that of a well-organized factory with optimal control and synchronization, with information and "materials" exchange between the agents (i.e., biochemical constituents) involved in living cycles prescribed by genes and abilities to react properly on the occurring internal and external changes. The behavior of living cells may be compared more favorably to patterns of narrative script than to an interactive mechanism of billiard balls in physics. Self-reproduction, ageing, and the supporting 
mechanisms for their existence make living cells an extremely complex theoretical object of research.

Fifty years ago, Brillouin presented three opinions from scientists concerning the application of the laws of physics and chemistry on living systems [2].

(a) Our present knowledge of physics and chemistry is practically complete, and these physical and chemical laws will soon enable us to explain life, without the intervention of any special life principles.

(b) We know a great deal about physics and chemistry, but it is presumptuous to pretend that we know all about them. We hope that, among the things yet to be discovered, some new laws and principles will be found that will give us an interpretation of life. We admit that life obeys all the laws of physics and chemistry at present known to us, but we definitely feel that something more is needed before we can understand life. Whether it is called a life principle or otherwise is immaterial.

(c) Life cannot be understood without reference to a life principle. The behavior of living organisms is completely different from that of inert matter. Our principles of thermodynamics, especially the second one, apply only to dead and inert objects: life is an exception to the second principle; a new life principle will have to explain conditions contrary to the second law of thermodynamics.

What if a "new life principle" should appear on the scientific scene, enabling us to treat living cells and organisms with as much success as we have enjoyed in atomic and solid state physics? New ideas on living systems are needed. Fifty years past since Brillouin publication definitely confirm the failure of the "optimists" presented by group (a) (see [8]).

Accordingly, we will focus on what has become quite popular: the recent presentation of living cells, organisms, and systems as networks of interconnected and interacting agents (chemicals, macromolecules, proteins, etc.). It is interesting to note that mathematicians and computer scientists initially hit on the idea of constructing and using artificial neural networks to perform complex intellectual computer simulations after observing the structure of the brain, which consists of interconnected living cells—-neurons. By giving the individual artificial neuron the simple, pure mathematics-based functions, it was possible to closely simulate the extremely complex behavior of the artificial network as a whole. This property, to generate complex behavior emerging from interconnected artificial neurons, finds wide practical application when a problem is complex and multicomponent and cannot be solved by traditional methods in a reasonable computer time. We also need to mention the chaos theory, which is introducing a new model of the dynamic behavior of complex systems. Ostensibly, the mathematical simulation of complex systems evidences an extreme sensitivity to internal and external infinitesimal influences. This property of chaotic behavior could be effectively used for mathematical modeling of information exchange within living objects: information exchange has an infinitely small impact compared to the energy exchange in inert matter, but could cause drastic reaction in the behavior of living systems. It is obvious that we are facing the extreme complexity of living systems dynamics. This is why the idea of using the paradigm of "biological artificial networks" as a theoretical tool for simulating individual living cells and systems 
finds supporters among biologists. It increasingly becomes an effective theoretical and experimental tool in the bio- and neurosciences $[1,7]$.

The question that we address in this paper relates to the possibility of constructing biological networks where each artificial agent will follow basic physicochemical laws. In addition to artificial neural networks, where individual neurons obey simple pure mathematics or logic-based rules, and to the abstract rules of cellular automata approach [9], we intend to "arm agents," namely, composed biological networks with specific constrains reflecting stoichiometry, the mechanism of biochemical reactions, mass conservation law, and the catalytical properties and information exchange of specific agents. We propose the way of formal introduction of "information exchange" into biochemical reactions dynamics. We expect that the use of known physicochemical principles and the introduction of new principles reflecting the specificity of living systems [4] will abet the interpolation as well as the extrapolation of observed experimental data, and will reduce the number of possible states generated by the artificial neural network to states with clear physicochemical sense.

\section{Background}

We present the living system as a set of $K$ modules. Each module will be considered as a multicomponent "biochemical reactor" composed by $A_{i}^{k}\left(i=1,2, \ldots, N_{k}\right)$ different biochemical constituents (agents) with $L$-space-distributed chemical reactions between them proceed by hypothesis about the mechanism expressed by the matrix of stoichiometric coefficients $v_{l i}^{k}: \sum v_{l i}^{k} A_{i}^{k}=0 ; l=1,2, \ldots, L^{k}$.

So, a living system could be presented as a network of interconnected modules and each module is simulated by the specific set of space-distributed biochemical reactions. For example, we suppose that in one module $(k=1)$ we have two chemical reactions $(L=2)$ between four agents $(N=4)$ :

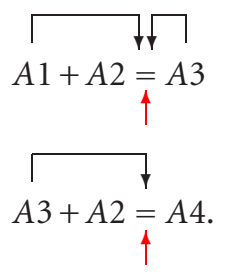

With black arrows, we mark the ability of a particular agent $A_{i}$ to affect the rate of the corresponding biochemical reaction "information exchange." Therefore, agents $A_{i}$ participate in biochemical transformations according to the chemical reaction mechanism, and have "signaling abilities" to regulate the reactions' corresponding rates. With red arrows, we mark the "signals" coming from other modules of the space-distributed agents.

Interaction between different modules could be introduced by additional reactions between elements from different modules as well as "information exchange" induced by the agents from $k$-module on the reaction rates in other modules.

For the mathematical model of the dynamics of $L$-space-distributed chemical reactions with "information exchange" for one module, we derived the basic equations from 
the extension of the maximum entropy principle and theory of dimensionality; the theory is called discrete chaotic dynamics (DCD) $[3,4]$ :

$$
\begin{gathered}
\prod X_{i}^{v l i}\left(t_{q}, \mathbf{r}\right)=\pi_{l}, \\
\sum \alpha_{i j} X_{i}\left(t_{q}, \mathbf{r}\right)=b_{j}, \\
\pi_{l}\left(X_{i}\left(t_{q-s}, \mathbf{r}^{\otimes}\right)\right)=\eta_{l} \exp \left\{-\left[\sum \delta_{l i} X_{i}\left(t_{q-s}\right)+\sum \beta_{l i} X_{i}\left(t_{q-s}, \mathbf{r}^{\otimes}\right)+\mathfrak{J}_{k}(\chi)\right]\right\},
\end{gathered}
$$

with initial and boundary conditions:

$$
\begin{aligned}
& X_{i}\left(t_{0}, \mathbf{r}\right)= \begin{cases}b_{j}, & i=1,2 \ldots, M, \\
0, & i=M+1, M+2, \ldots, N\end{cases} \\
& X_{i}\left(t_{q}, \mathbf{r}\right)= \begin{cases}X_{i}\left(t_{q}, \mathbf{r}=R\right), & \text { inside the considered discrete space } R, \\
0, & \text { outside the considered discrete space. }\end{cases}
\end{aligned}
$$

Here, $X_{i}\left(t_{q}, \mathbf{r}\right)$ are the concentrations of constituents $A_{i}, \alpha_{l i}$-molecular matrix presents a number of system components, $j=1,2, \ldots, M$ in ith constituent (agent), and $b_{j}=$ total amount (concentration) of $j$ th component.

Matrix $v_{l i}$ (given initial hypothesis about mechanism of biochemical reactions) could be presented in the following form, with $G, F, J, K$ submatrixes and $G$-nondegenerate ma$\operatorname{trix}(M \times M)$ :

$$
v_{l i}^{T}=\left|\begin{array}{cc}
G & F \\
J & K
\end{array}\right| .
$$

Under these conditions, matrix $\alpha_{i j}$ has the following expression:

$$
\alpha_{i j}^{T}=\left|-J x G^{-1} \quad I\right|
$$

$\mathbf{r}$ is the $3 \mathrm{D}$ discrete space coordinates for designating $\mathfrak{R}$ space-distributed microvolumes, $t_{q}$ denotes a discrete sequence of the system evolution, where $q=1,2, \ldots$; and $s=1,2, \ldots$, denotes previous to $t_{q}$ system states. For example, $s=1$ means that we are considering just previous to the current system state, $t_{q-1}$; the state when $s=2$ means system state separated by two evolution steps from the current system state, and so forth. $X_{i}\left(t_{q-s}, \mathbf{r}^{\otimes}\right)$ are $N$ concentrations calculated according to (2.2), (2.3), and (2.4) when systems in the states $t_{q-s}$ and $X_{i}\left(t_{q-s}, \mathbf{r}^{\otimes}\right)$ are taken from the neighbored positions, denoted as $\mathbf{r}^{\otimes}$, to the currently considered concentrations $X_{i}\left(t_{q}, \mathbf{r}\right)$. The $\delta_{l i}$ empirical parameters characterize "information exchange" between the agents from the state $q-s$ by their influence on the rate of reaction $l$ which happens at coordinates $\mathbf{r}$, and the $\beta_{l i}$ parameters characterize influences on the reaction $l$ provided by the neighbored agents with coordinates $\mathbf{r}^{\otimes}$. On [5, Figure 1], we present one module of the $2 \mathrm{D}$ biological network which consists of nine 
space-distributed "microvolumes" with discrete coordinates $\mathbf{r}(1,1 ; 1,2 ; 1,3 ; 2,1 ; 2,2 ; \ldots)$. In each element of our network, we have $N$ constituents with $L$ reactions with "information exchange." Each of these reactions depended on all the other space-distributed agents. Parameters $\beta_{l i}$ characterize the strength of these connections and their impact on the dynamics of the systems. Other modules could be included in this network just by additional intermodular agents reactions (which increases the number of agents $N$ ) with intermodular information exchange (by adding additional arrows coming from different modules). In that case, we need to introduce a function $\mathfrak{I}_{k}\left(\chi_{k}, X_{v}^{k}\right)$ with corresponding parameters $\chi_{v}$ and concentrations $X_{v}^{k}(v=1,2, \ldots, V)$ composing other modules into (2.4), where $K$ is a number of agents composing module $k$ (see [5, Figure 1]).

Our proposed system of nonlinear difference equations (2.2), (2.3), and (2.4) corresponds to the extended on open and far from equilibrium conditions principle of maximum of entropy or minimum of free energy. This new principle conveys the idea that systems evolution proceeds through the discrete systems states in such a way that for any given mechanism of biochemical interactions and scheme of "information exchange" the following function reaches its minimum in ND concentration space $X_{i}\left(t_{p}, \mathbf{r}\right)$ :

$$
\min \Theta\left(X_{i}\left(t_{p}, \mathbf{r}\right)\right)=\sum X_{i}\left(t_{p}, \mathbf{r}\right)\left\{\ln X_{i}\left(t_{p}, \mathbf{r}\right)+\ln \pi_{l}\left(X_{i}\left(t_{q-s}, \mathbf{r}^{\otimes}\right)\right)\right\},
$$

under the constrains (2.3).

The mathematical structures of the difference equations we present do not include any form of time, neither classical continuous astronomic time, nor the so-called "discrete time" that has no clear meaning. Nevertheless, using difference equations we can simulate the system evolution by calculating changes of the agents' concentrations according to (2.2), (2.3), and (2.4). These changes are the results of interactions between the constituents on two levels: on the level of biochemical transformations according to the biochemical reactions, and on the level of control of these reactions by "information exchange." This control allows delaying or even freezing $\left(\delta_{l i}, \beta_{l i}=0\right)$ some reaction, or contrariwise, accelerating or catalyzing it. Space-distributed interactions play an essential role in our network and reflect the real situation with living systems when structural complexity and space nonhomogeneity of the dynamic processes in the living cells depend on space-distributed interactions. The space of living systems is hardly comparable to the Euclidian 3D space where we can place and analyze the trajectories of systems. Living systems, by their appearance and in their development, create a variety of forms and complex objects (patterns), instead of trajectories in the mechanical sense, or as understood in classical dynamics. The modeling of the processes, which will bring us to the simulation of the appearance of the biological forms similar to the experimentallyobserved living objects, is one of the main goals of the artificial biological networks.

The proposed mathematical model and the artificial biological networks will be instrumental to calculate the sequences or the evolution of the systems states. When we relate this dynamics to the concrete constituents of the systems, and their interactions and signaling abilities (information exchange), our proposed mathematical model will work as a photo camera giving instant pictures of space-distributed concentration of the agents. Putting these pictures into a sequence, we will have on film the evolution of living systems. What precisely is the time when we create this movie record? Is it important 


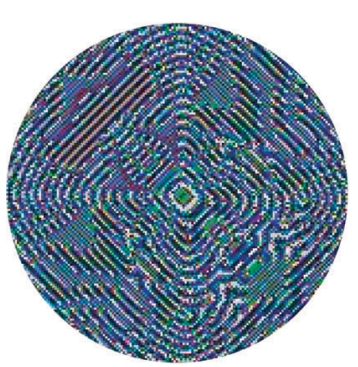

1

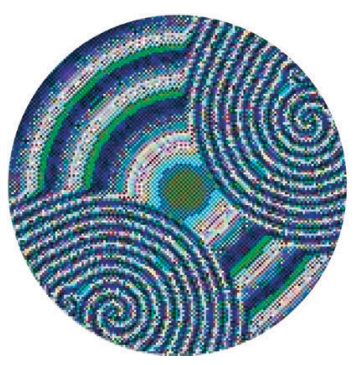

4

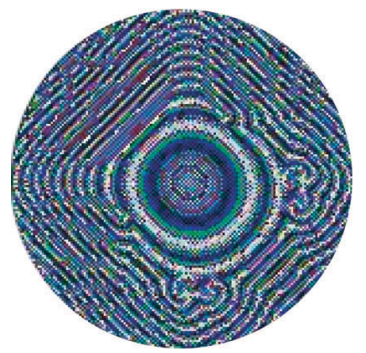

2

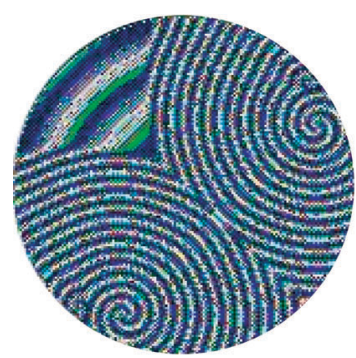

5

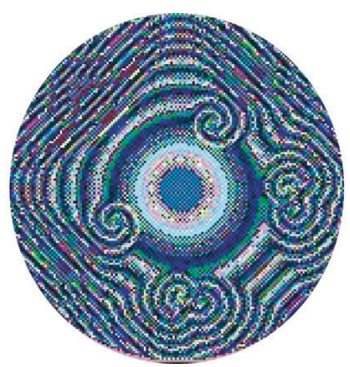

3

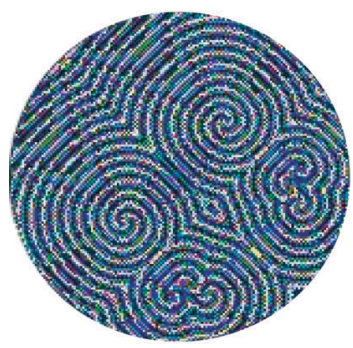

6

Figure 3.1

to discuss astronomic time to understand the dynamics of living systems? In five minutes of movie time, we can cover the hall life of the participants. The types of interaction and transformation (mechanisms of discrete space-distributed reactions) that "information exchange" accomplishes define the living systems dynamics. Presented by timeless dynamics, the evolution of living systems could then be related to the traditional astronomic time scale; continuous presentation could interpolate discrete space coordinates. The parameters presented in (2.2), (2.3), and (2.4) are the subject of statistical analysis of corresponding experimental data on concrete system dynamics.

\section{Some results of numerical simulations}

To demonstrate the facilities of the proposed theoretical approach and some of the resulting basic equations, we performed some numerical simulations for some mechanisms of the biochemical transformations with information exchange. In our example, we consider one module $(k=1)$, using 2D space system $(R=2)$. We constrained the system "memory" just by one previous state $(s=1)$, and with "information exchange" between the agents of the closest neighbors. The mechanisms of biochemical reactions in all discrete space-distributed "microvolumes" $(160 \times 160)$ are the same and all have four agents 


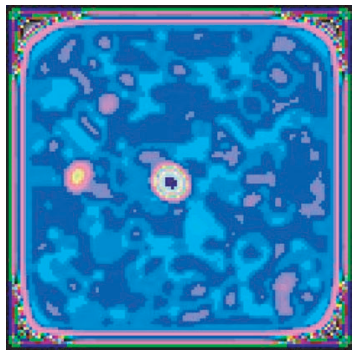

1

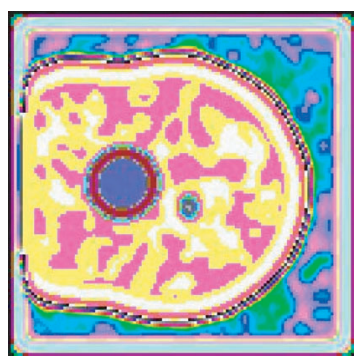

4

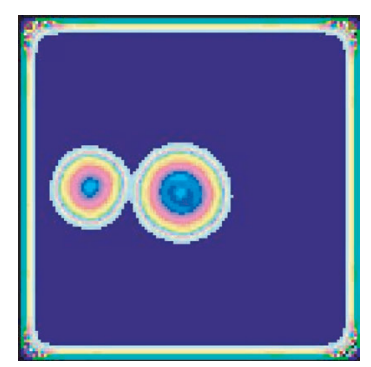

2

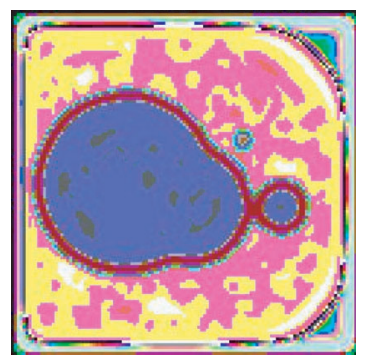

5

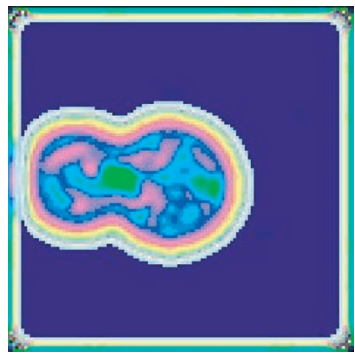

3

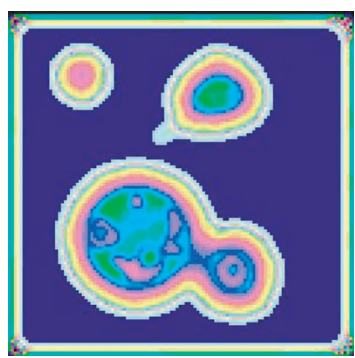

6

Figure 3.2

$A 1, A 2, A 3$, and $A 4$ :

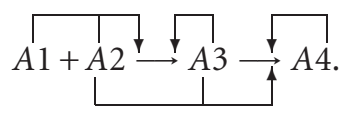

On the diagrams you can see in color the concentration of the evolution of the agent $A_{1}\left(X_{1}\right)$ distributed among $160 \times 160$ microvolumes, simulated by (2.2), (2.3), and (2.4), written for the particular mechanism of biochemical transformations, starting from the initial conditions (2.5a) and satisfying the boundary conditions (2.5b). All concentrations are normalized $0<X_{i}\left(t_{p}, \mathbf{r}\right)<1$ with $1 / 256$ resolution. Therefore, each calculated value $X_{i}\left(t_{p}, \mathbf{r}\right)$ could be presented by the particular color from the 256-colored palette. On Figure 3.1, you can observe six system states for (1) $q=50$, (2) $q=100, \ldots$, (6) $q=300$. We can observe dynamics of spiral and ring waves formation and evolution in the form of distribution of $X_{1}$ concentration. Even in the simple mechanism of interaction of agents (3.1), and the use of discrete chaotic dynamics equations (2.2) and (2.3), the space-distributed patterns obtained show the process of complex organization, as in the $\mathrm{BZ}$ reaction (see sea shells, and so forth) already noticed. Changing the parameters $\eta_{l}, \delta_{l i}, \beta_{l i}$, and $b j$, we can simulate a variety of different regimes of spiral and ring wave 


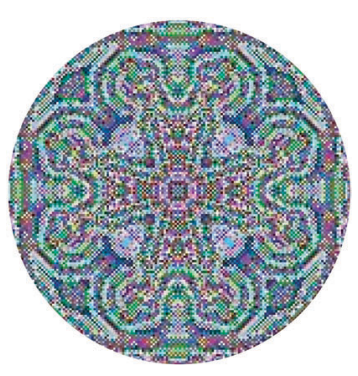

1

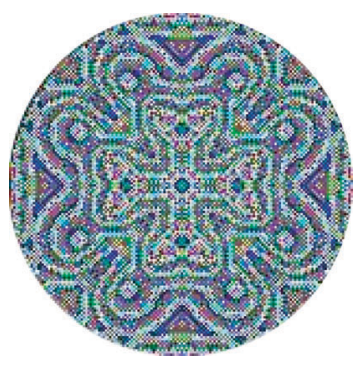

4

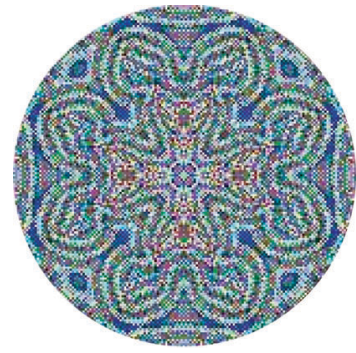

2

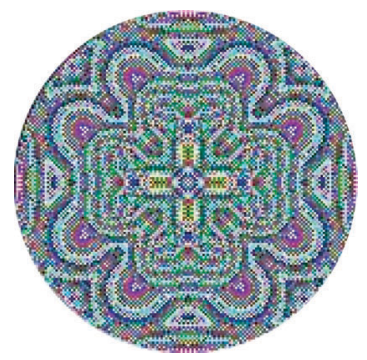

5

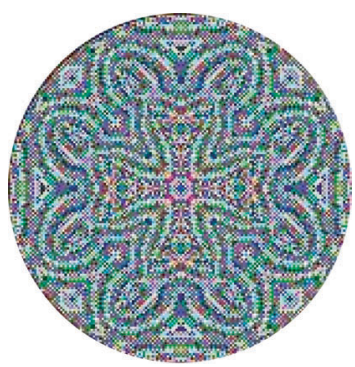

3

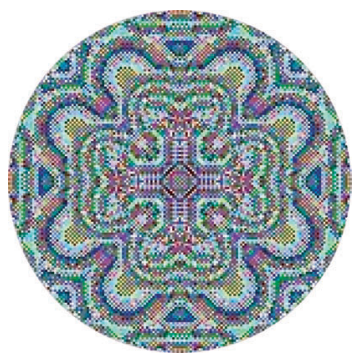

6

Figure 3.3

formations. Thus, we can closely correspond to the experimental data and make some predictions on regimes yet unobserved.

By changing the mechanism of reactions (3.1) to

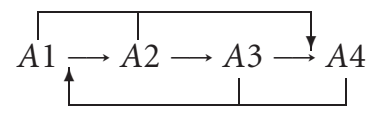

and by adjusting the parameters of (2.2), (2.3), and (2.4) written for (3.2), we can generate another type of the patterns presented in Figure 3.2. These are just a few examples of the ability of the proposed mathematical model to simulate the formation of complex patterns and their spatial-temporal evolution. The pattern formation dynamics we have presented apparently indicate a pattern of coordinated behavior from all the agents involved in biochemical transformation with "information exchange." From the initial state of equal distribution of concentration of agents among the microvolumes, the whole system shows patterns when agent concentrations form some sort of order (spirals, rings, strings, and of other complex patterns on the scale of the whole system) as a result of interactions with information exchange between the constituents. Figure 3.3 
presents artistic patterns in mandala form. If we will look at our network as $160 \times 160$ interconnected neurons with biochemical reactions inside each neuron with "information exchange" between them, we can relate different patterns generated by our network to the brain creativity processes in mandala form. According to Jung, mandalas as psychological phenomena appear spontaneously in dreams, in certain states of conflict, and in cases of schizophrenia [6]. The fact that proposed mathematical model could be used to generate patterns in a form similar to Jung's mandalas opens the way for theoretical and experimental studies of brain functioning, including the study of differentiated abnormalities such as schizophrenia and others [5].

\section{Conclusions}

Artificial brain systems [5] as well as artificial life systems based on the presented theoretical approach could serve as theoretical tools to increase our understanding of the internal biochemical mechanisms accomplished with information exchange. These interactions are responsible for the dynamics of living and thinking systems such as those that appear from nonliving constituents. The introduction of a new hypothesis, namely, of a general extreme principle responsible for living systems evolution (2.8), challenges us to discover the laws of nature that drive us. It is hard to overestimate the role of the "information exchange" that naturally appeared in the proposed paradigm followed by concrete mathematical equations. The meaning of "information exchange" used in DCD is different from the classical attempts to define information as a measure of systems order, or by number of symbols needed to change the systems state. Our meaning of "information exchange" is a dynamic category reflecting the processes of a special type of interaction of systems constituents unlike and directly contrary to energy exchange (energy can be transmitted to the receiver without any special condition). "Information exchange" could appear only if both participants (transmitter and receiver) of the dynamic processes are ready for it. In that case, even an infinitely small signal (in a sense of the energy contained) from the transmitter could drastically change state of the receiver. The patterns generated by DCD algorithms could serve to measure the effects of "information exchange" introduced by the presented mathematical model. "Information exchange" in complex and living systems opens the possibility of employing the chaos theory principles and the related mathematical tools. This is why the proposed difference equations (2.2), (2.3), and (2.4), which contain numerous chaotic states, could be used for mathematical modeling of the "information exchange" in living systems. Chaotic regimes synchronization, emergent behavior, and complex systems self-organization are the main results from chaotic systems research. They could be widely used for our purposes when combined with the laws of nature introduced in basic equations derived for DCD of living and thinking systems. The use of chaotic regimes generated by the concrete equations reject the idea of introducing probabilities into the basic equations, because observed complex living systems dynamics could be simulated and explained by the use of deterministic equations (2.2), (2.3), and (2.4). New and different structures and regimes that spontaneously appear are due to the extreme complexity of deterministic chaotic solutions; they reflect the biochemical transformations of real systems, their information exchange, and their ability to memorize previous systems states and to follow general dynamic principles. In 
this context, mutations as well as new constructive thoughts (innovations) could appear in living and thinking systems as the result of the biochemical reaction that information exchange catalyzes. This could be considered as a source for creating new patterns and species.

The idea of using biological networks operating under defined laws of nature and basic equations in the form and manner of difference equations is an attempt to fight the extreme complexity of the object of investigation, by first using difference equations as a new calculus for basic laws of nature. The opposite problem is to introduce into these equations the empirical parameters and concrete architecture of the networks for the particular living or thinking system. This is as important as the proposed mathematical model itself. The solution of this polar problem could only come from experimental studies of the concrete living dynamical system with further statistical analyses of collected experimental data. That will correct the initial hypothesis on the mechanism of the interaction of the constituents of the system; it will abet defining the connections of the system network and will give concrete values to the mathematical model parameters. This research will also help in the creation of artificial living and thinking systems with the ability to perform numerous functions with the efficiency of human beings and the speed of electronic computers calculations. We are barely at the threshold of discovering the specific laws of nature responsible for the dynamics of living and thinking systems. However, it is hard to expect any great advance in the research of living systems without comparable progress in theoretical studies as in experimental biology and neurosciences in the past. We hope that the proposed theoretical approach for constructing complex biological networks, namely, mathematically modeling of the dynamics of complex living and thinking systems, but using the discourse biochemists on multicomponent reactions mechanisms, will allow the introduction of a new general principle responsible for different kind of living agents interaction with information exchange. The use of difference equations for performing computer simulations will allow us to better understand the principles of life. For medicine, this means the creation of hybrid "human-artificial life" systems with tremendous new facilities. They can lead to the invention of a new generation of artificial brain systems instrumental in solving extremely complex problems in science, technology, economy, and medicine.

\section{Acknowledgments}

I would like to express my gratitude to the Department of Economy and School of Engineering at the University of Southern California for being my host during sabbatical and providing me with various communications with the faculty, and in particular to the USC Professors R. H. Day, F. Udwadia, and M. Arbib. This paper is dedicated to the memory of the great scientist and man, Professor Ilya Prigogine, to whom I am personally obliged for many innovative ideas and especially for the scientific spirit he generated during his life.

\section{References}

[1] U. Alon, Biological networks: the tinkerer as an engineer, Science 301 (2003), no. 5641, 18661867.

[2] L. Brillouin, Life, thermodynamics, and cybernetics, Amer. Sci. 37 (1949), 554-568. 
[3] V. Gontar, New theoretical approach of physico-chemical reactions dynamics with chaotic behavior, Chaos in Chemistry and Biochemistry (R. J. Field and L. Gyorgyi, eds.), World Scientific, London, 1993, pp. 225-247.

[4] Discrete dynamics for mathematical simulation of living systems, Chaos Solitons Fractals 8 (1997), no. 4, 517-524.

[5] _ Theoretical foundation for Jung's "Mandala Symbolism" based on discrete chaotic dynamics of interacting neurons, Discrete Dyn. Nat. Soc. 5 (2000), no. 1, 19-28.

[6] C. G. Jung, Mandala Symbolism, Princeton University Press, New Jersey, 1972.

[7] H. H. McAdams and L. Shapiro, A bacterial cell-cycle regulatory network operating in time and space, Science 301 (2003), no. 5641, 1874-1877.

[8] I. Prigogine, The End of Certainty: Time, Chaos, and the New Laws of Nature, The Free Press, 1997.

[9] S. Wolfram, A New Kind of Science, Wolfram Media, Illinois, 2002.

V. Gontar: International Group for Chaos Studies, Ben-Gurion University of the Negev, P.O. Box 653, 1 Hashalom, Beer-Sheva 84105, Israel

E-mail address: galita@bgumail.bgu.ac.il 


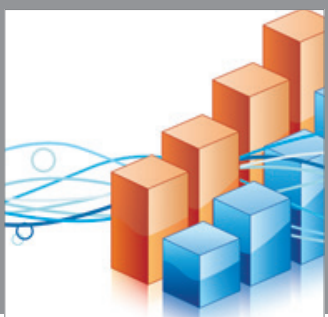

Advances in

Operations Research

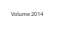

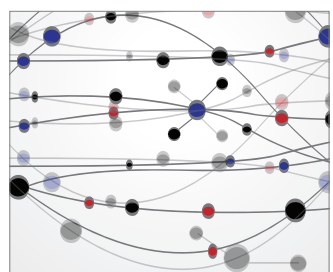

\section{The Scientific} World Journal
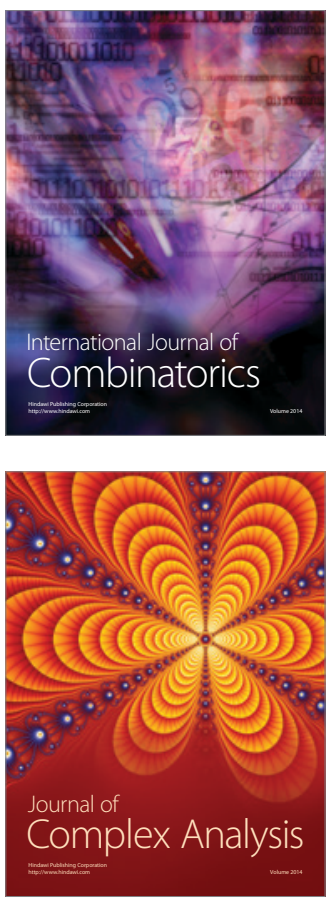

International Journal of

Mathematics and

Mathematical

Sciences
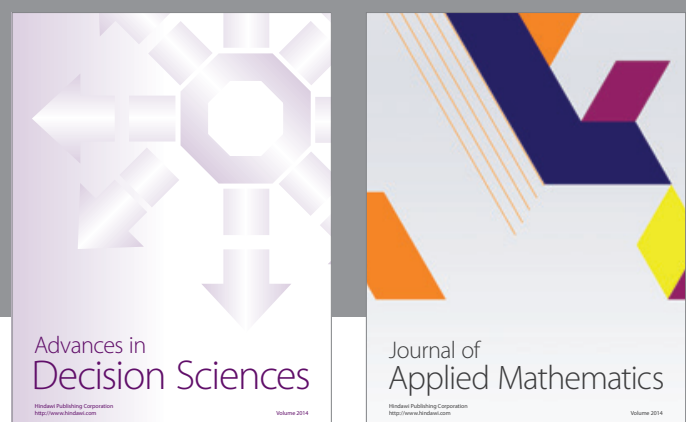

Journal of

Applied Mathematics
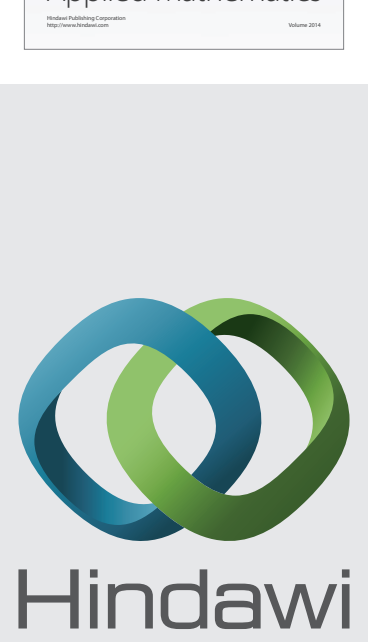

Submit your manuscripts at http://www.hindawi.com
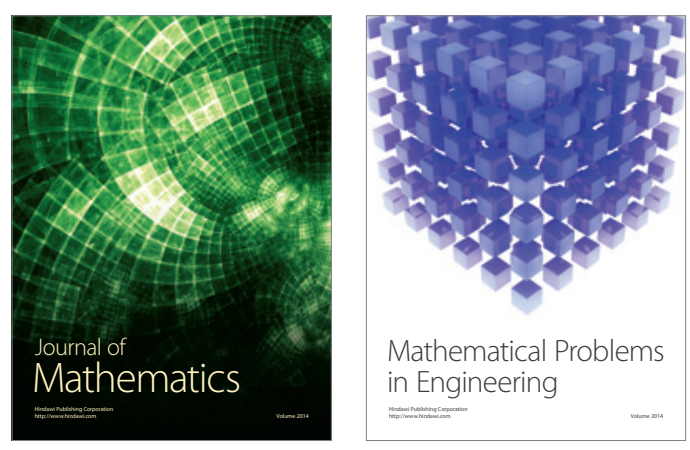

Mathematical Problems in Engineering
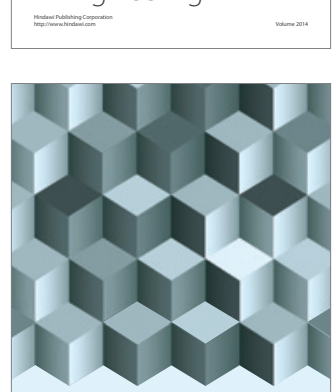

Journal of

Function Spaces
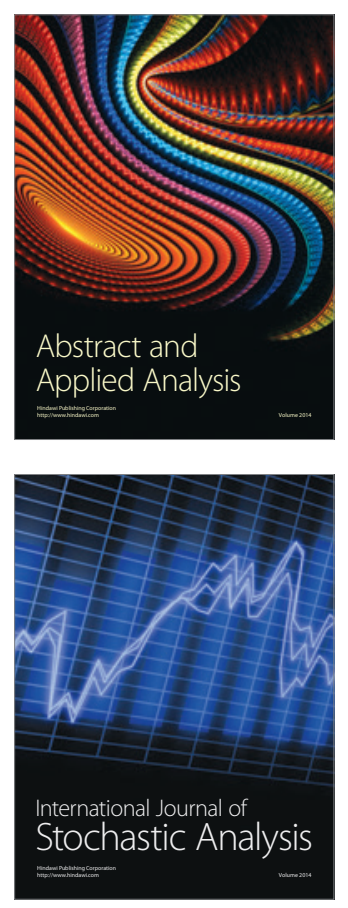

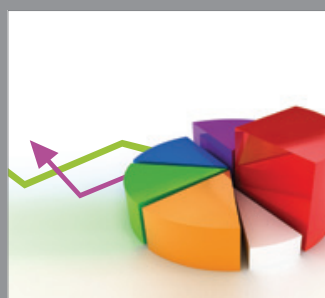

ournal of

Probability and Statistics

Promensencen
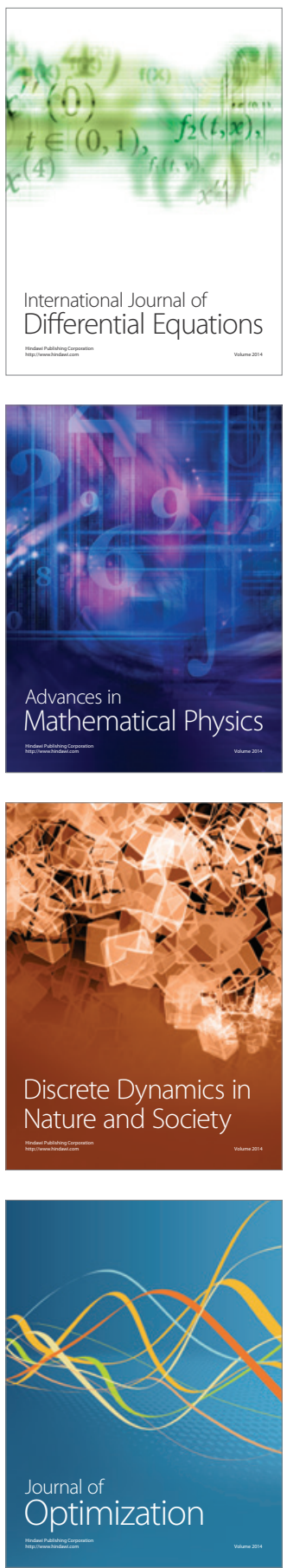